\title{
High expression of IL-9R promotes the progression of human hepatocellular carcinoma and indicates a poor clinical outcome
}

\author{
HONG-JUN LI ${ }^{1 *}$, QI-MAN SUN ${ }^{2 *}$, LONG-ZI LIU $^{2 *}$, JUN ZHANG $^{1}$, JUN HUANG $^{1}$, \\ CHENG-HONG WANG ${ }^{1}$, RUI DING ${ }^{1}, \mathrm{KANG} \mathrm{SONG}^{2}$ and $\mathrm{ZHONG} \mathrm{TONG}{ }^{1}$ \\ ${ }^{1}$ Department of Hepatobiliary Surgery, The First People's Hospital of Hefei, Anhui 230000; \\ ${ }^{2}$ Liver Cancer Institute, Zhongshan Hospital, Fudan University, Shanghai 200032, P.R. China
}

Received March 18, 2015; Accepted May 16, 2015

DOI: $10.3892 / o r .2015 .4060$

\begin{abstract}
Interleukin-9 receptor (IL-9R) overexpression has a pivotal role in human hematological malignancies. However, the expression of IL-9R and its biological role in human solid tumors remains elusive. In the present study, western blot analysis and RT-qPCR were used to determine the expression of IL-9R in hepatocellular carcinoma (HCC) cell lines and tumor tissues. Proliferation, cell cycle, apoptosis and Transwell assays were used to examine the biological role of IL-9R in HCC cells. The results showed that IL-9R and its ligand IL-9 were constitutively expressed in HCC cells and tissues. Moreover, the expression levels of IL-9R and IL-9 were significantly higher in tumor tissues compared to the peritumor liver tissues. Functional experiments suggested that IL-9R significantly promoted HCC cell proliferation, invasion and inhibited apoptosis, possibly by acting through the IL-9/IL-9R axis. After silencing IL-9R, the expression of VEGF, p-p38, p-STAT3 and MMP9, markedly decreased suggesting the potential involvement of these molecules in IL-9R activity. Immunohistochemistry-based survival analysis revealed that a differential expression of IL-9R in HCC tissue was a significant and independent prognostic factor for survival [HR, 1.66; 95\% confidence interval $(\mathrm{CI}), 1.17-2.36 ; \mathrm{P}=0.005]$ and recurrence [HR, 1.50; 95\% CI, 1.04-2.17; P=0.03]. In addition, a high IL-9R expression positively and significantly correlated with larger $(\mathrm{P}=0.012)$ and advanced tumor stage $(\mathrm{P}=0.018)$. The findings indicated that IL-9R was constitutively expressed and exerted
\end{abstract}

Correspondence to: Dr Zhong Tong, Department of Hepatobiliary Surgery, The First People's Hospital of Hefei City, 390 Huaihe Road Hefei, Anhui 230000, P.R. China

E-mail: toto6993@163.com

Dr Kang Song, Liver Cancer Institute, Zhongshan Hospital, Fudan University, 180 Fenglin Road, Shanghai 200032, P.R. China

E-mail: song.kang@zs-hospital.sh.cn

${ }^{*}$ Contributed equally

Key words: hepatocellular carcinoma, interleukin-9 receptor, tumorigenesis, MAPK, STAT3 a tumor-promoting effect in HCC, whose expression level may be a useful biomarker of tumor invasiveness and patient clinical outcome.

\section{Introduction}

Hepatocellular carcinoma (HCC) is one of most frequently occurring neoplasms worldwide (1) and a major cause of cancer-associated mortality owing to its high potential of invasion and metastasis. Surgery, including liver resection and transplantation, remains the most effective treatment for HCC. However, high recurrence or metastatic rate after surgery hinders further improvements in HCC survival (2). Molecules involved in HCC recurrence and metastasis are potential factors predicting patient outcome, and personalized treatment depends mostly on the increased use of diagnostic markers for treatment allocation $(3,4)$. Therefore, the identificaiton of an effective biomarker is pivotal in screening cancer, assessing general population risk, evaluating treatment-response and monitoring recurrence.

Interleukin 9 (IL-9) cytokine is a 14-kDa glycoprotein comprising 144 amino acids (5). The receptor of IL-9 (IL-9R) is a member of hematopoietin consisting of two subunits, the IL-9 specific $\alpha$-chain receptor and a member of the $\gamma$-chain-receptor family commonly shared with IL-2, IL-4, IL-15 and IL-17 (6-8). IL-9 was considered to be a T-lymphocyte growth factor and a cytokine produced by the Th2 subset $(6,9)$. As indicated by the T-cell growth function of IL-9, IL-9R is expressed in T cells and effector T cells (10). Addtionally, IL-9R is highly expressed in the Th2 and Th17 cell subsets (8). However, in asthma patients, IL-9R was reported to be expressed in mast and polymorphonuclear cells $(7,8)$. The secretion of IL-9 was observed in a variety of immune cells, including Th2, Th9, Th17 and Treg cells, NKT cells and mast cells (5). Activation of IL-9R results in phosphorylation of Jak1 in the $\alpha$ chain and Jak3 in the $\gamma$ chain, which leads to the downstream activation of STAT3, MAPK and PI3K pathways $(8,11,12)$.

IL-9 and its receptor have been investigated in bronchial asthma (13), autoimmune diseases (14), parasitic infections (15) and atopic dermatitis (16). In the mouse inflammatory models, IL-9 showed proinflammatory activity and blocking IL-9 inhibited airway remodeling in chronic lung 
inflammation (17). In chronic lymphocytic leukemia (CLL), IL-9R overexpression inhibited apoptosis significantly (18). Aberrant expression of IL-9R was also observed in several types of human leukemia (19).

HCC is a well-known inflammation-related cancer (20). However, the expression of IL-9R and its biological role in HCC tumorigenesis and metastasis remain largely unknown. In the present study, we identified aberrant IL-9R expression in HCC and investigated its biological role in HCC cells as well as the clinical significance in $329 \mathrm{HCC}$ patients undergoing resection. A high expression of IL-9R in HCC correlated with tumor aggressiveness and served as an independent prognostic factor for HCC patients.

\section{Materials and methods}

Patient samples. We randomly selected $12 \mathrm{HCC}$ tissue samples for RT-qPCR analysis from the patients who underwent liver resection at the Zhongshan Hospital, Fudan University (Shanghai, China). Samples were collected within $15 \mathrm{~min}$ of hepatectomy, delivered in liquid nitrogen and stored at $-80^{\circ} \mathrm{C}$. Tumor specimens used in the tissue microarray analysis were consecutively selected from 329 HCC patients, who underwent liver resection in the Liver Cancer Institute, Zhongshan Hospital, Fudan University in 2006. After fixed in $4 \%$ formaldehyde for $24 \mathrm{~h}$, tissues of HCC patients were used for construction of paraffin-embedded blocks as described previously (21). The clinicopathological characteristics of the patients are shown in Table I.

Detailed patient inclusion and exclusion criteria have been previously described (21). The tumor stage was assessed according to the Barcelona Clinic Liver Cancer (BCLC) staging system (22) and the tumor-node-metastasis (TNM) system of the International Union against Cancer (UICC, 7th edition). Tumor differentiation was graded using the Edmondson grading system. Detailed procedures for postoperative surveillance and treatment modalities have been previously described (23). The present study was approved by the Research Ethics Committee of Zhongshan Hospital (Shanghai, China). Written consent was provided by the patients. Overall survival (OS) was defined as the date of surgery and death or the last observation recorded. Relapse-free survival (RFS) was defined as the interval between resection and tumor recurrence, death or the last follow up. The data were censored for surviving patients and patients without signs of recurrence.

Cell lines. Six human HCC cell lines were used in the present study: MHCC97H, MHCC97L (Liver Cancer Institute, Fudan University, Shanghai, China), PLC/PRF/5 (Japanese Cancer Research Bank, Tokyo, Japan), HepG2, Hep3B (American Type Culture Collection, Manassas, VA, USA) and the immortalized human normal L-02 liver cell line (Cell Bank, Chinese Academy of Sciences, China). The cell lines were routinely maintained in DMEM or RPMI-1640 supplemented with $10 \%$ heat-inactivated fetal bovine serum, $100 \mathrm{U} / \mathrm{ml}$ penicillin and $100 \mathrm{mg} / \mathrm{ml}$ streptomycin at $37^{\circ} \mathrm{C}$ in a humidified incubator under $5 \% \mathrm{CO}_{2}$.

RNA isolation and RT-qPCR. Total RNA was extracted from cell lines and frozen tumor specimens using TRIzol reagent
(Invitrogen, Carlsbad, CA, USA) according to the manufacturer's instructions. mRNA expression of IL-9 and IL-9R in HCC cell lines and tumor tissues was detected by RT-qPCR using an ABI7900HT (Applied Biosystems, Foster, CA, USA). RT-qPCR was performed using a SYBR PrimeScript RT-PCR kit (Takara Bio Inc., Otsu, Shiga, Japan) according to the manufacturer's instructions. The primer sequences used were: IL-9 forward, 5'-CTCTGCCCTGCTCCTGTGCT-3' and reverse, 5'-GCCTGCCGTGGTTTGGTT-3'; IL-9R forward, 5'-TCACCATCACTTTCCACCAC-3' and reverse, 5'-CCT CTACCACATCATCCTCC-3'; GAPDH forward, 5'-CGGAGT CAACGGATTTGGTCGTAT-3' and reverse, 5'-AGCCTT CTCCATGGTGGTGAAGAC-3'. Relative mRNA levels were detected based on the $\mathrm{Ct}$ values, and corrected to GAPDH expression, according to the $2^{-\Delta \mathrm{Ct}}$ method. The experiments were performed in triplicate.

Western blot analysis. The protein level expression of IL-9R and signaling molecules was detected by western blot analysis. Briefly, the cells were washed twice with ice-cold PBS and frozen tumor specimens were ground under liquid nitrogen. Total protein was extracted in lysis buffer for $45 \mathrm{~min}$ on ice. Equal amounts of protein were separated by $10 \%$ SDS-PAGE and transferred to a PVDF membrane (Millipore, Billerica, MA, USA) using a mini trans-blot apparatus (Bio-Rad Laboratories, CA, USA). The membrane was blocked with PBSwith $0.05 \%$ Tween-20 containing 5\% non-fat dry milk for $1 \mathrm{~h}$ and incubated for $2 \mathrm{~h}$ at room temperature with monoclonal/polyclonal mouse/rabbit anti-human antibodies including IL9R (Abcam, Cambridge, MA, USA), VEGF, p-JNK, JNK, p-STAT3, STAT3, p-STAT5, STAT5, p38, p-p38, p-ERK, ERK, MMP9 (Cell Signaling, Danvers, MA, USA) and GADPH (SigmaAldrich, St. Louis, MO, USA). Each experiment was repeated at least three times.

SiRNA-mediatedIL-9Rsilencing.AnAnnexin V-FITCapoptosis detection kit was used to determine IL-9R knockdown-mediated effects on cell apoptosis. The target siRNA sequence for IL9R used was: 5'-CCTCTCCAGCGATGTTCTT-3'. MHCC97H and MHCC97L were used in the siRNA analysis and transfection of siRNA was carried out using Lipofectamine 2000 (Invitrogen) according to the manufacturer's instructions .

Cell proliferation, cycle, invasion and apoptosis assays. Cell proliferation was analyzed using a Cell Counting kit-8 (CCK-8) assay kit (Dojindo Corp, Japan). The Tecan Infinite 200 microplate reader (Tecan Inc., Mäennedorf, Switzerland) was used to detect absorbance at $450 \mathrm{~nm}$.

Transwell ( $8-\mu \mathrm{m}$ pore size; Millipore) coated with Matrigel (BD Biosciences, San Jose, CA, USA) was used to evaluate cell invasion. Cells $\left(1 \times 10^{5}\right)$ were added to the upper chamber and after 48-h incubation, the cells remaining in the upper chamber were removed using cotton swabs. The cells that had migrated or invaded were fixed and stained in a dye solution containing $0.1 \%$ crystal violet and $20 \%$ methanol. The cells were counted in five random fields at a magnification of $\mathrm{x} 100$. The experiments were performed in triplicate.

Cell cycle and apoptosis were analyzed using flow cytometry. Briefly, the cells were collected, stained and fixed in ice-cold 70\% ethanol for FACS analysis (Beckman-Coulter, 
Table I. Correlation between IL-9R expression and clinicopathological characteristics in HCC patients.

\begin{tabular}{|c|c|c|c|}
\hline Variables & IL-9R high & IL-9R low & P-value \\
\hline Age, years $(\leq 52 />52)$ & $37 / 47$ & $125 / 120$ & 0.270 \\
\hline Gender (male/female) & $73 / 11$ & $205 / 40$ & 0.480 \\
\hline Hepatitis history (yes/no) & $74 / 10$ & $218 / 27$ & 0.825 \\
\hline Liver cirrhosis (yes/no) & $71 / 13$ & $199 / 46$ & 0.496 \\
\hline $\operatorname{AFP}(\leq 20 />20 \mathrm{ng} / \mathrm{ml})$ & $36 / 48$ & $92 / 153$ & 0.389 \\
\hline $\operatorname{ALT}(\leq 75 />75 \mathrm{U} / 1)$ & $67 / 17$ & $210 / 35$ & 0.197 \\
\hline$\gamma-\mathrm{GT}(\leq 54 />54 \mathrm{U} / 1)$ & $25 / 59$ & $52 / 193$ & 0.111 \\
\hline Tumor size $(<5 / \geq 5 \mathrm{~cm})$ & $34 / 50$ & $138 / 107$ & 0.012 \\
\hline \multicolumn{4}{|l|}{ Tumor number } \\
\hline (Single /multiple) & $66 / 18$ & $209 / 36$ & 0.150 \\
\hline Tumor capsule (yes/no) & $51 / 33$ & $128 / 117$ & 0.179 \\
\hline \multicolumn{4}{|l|}{ Tumor differentiation } \\
\hline (I-II/III-IV) & $62 / 22$ & $176 / 69$ & 0.727 \\
\hline Vascular invasion (yes/no) & $28 / 56$ & $77 / 168$ & 0.747 \\
\hline TNM stage (I/II-III) & $39 / 45$ & $148 / 97$ & 0.018 \\
\hline BCLC stage (0-A/B-C) & $37 / 47$ & $105 / 140$ & 0.849 \\
\hline
\end{tabular}

Chi-square test was used for comparison of groups. AFP, $\alpha$-fetoprotein; ALT, alanine aminotransferase; $\gamma$-GT, gamma-glutamyl transferase; TNM, tumor-node-metastasis; BCLC, Barcelona clinic liver cancer.

Brea, CA, USA) using MultiCycle AV for Windows 5.0 (Phoenix Flow Systems, San Diego, CA, USA). Apoptosis was measured using apoptosis detection kit (BD Biosciences).

Tissue microarray and immunohistochemistry. Tissue microarray blocks were constructed as previously described (23). Briefly, the HCC tissues were reviewed by two histopathologists and representative tumor areas free from necrotic and hemorrhagic materials were premarked in the paraffin blocks. Two core biopsies of $1 \mathrm{~mm}$ in diameter were obtained from the tumor and peritumor region of the donor blocks and transferred to the recipient paraffin block at defined array positions. Four different tissue microarray blocks were then constructed. Immunohistochemistry was performed according to a previously described two-step protocol (Novocastra, Newcastle, UK) (23). The primary antibody used in immunohistochemistry was rabbit polyclonal anti-human IL-9R (Abcam, Cambridge, UK).

Immunohistochemical staining was assessed by two independent pathologists without knowledge of patient characteristics. For IL-9R staining, we determined the percentage of cells with a positive score in the cytosol or membrane in the whole biopsy cylinder. The scoring procedure was as follows: the staining intensity was first scored $(0$, negative; 1 , weak; 2 , moderate; 3 , high) and the percentage of positive cells was scored $(0,0 \%$ positive cells; $1,1-10 \%$ positive cells; $2,11-50 \%$ positive cells; $3,>50 \%$ positive cells). The final score was calculated by multiplying the staining intensity score by the percentage of positive cells. Samples were defined as negative when the final scores were 0-3 and positive when 4-9.

Statistical analysis. Statistical analysis was performed using SPSS 18.0 for windows (SPSS, IBM, NY, USA). Survival analysis was performed by the Kaplan-Meier method and the log-rank test. Univariate and multivariate analyses were based on the Cox proportional hazards regression model. The Chi-square test, Fisher's exact probability and Student's t-test were used for comparison between groups. The experimental data were presented as means $\pm \mathrm{SD}$. A two-tailed $\mathrm{P}<0.05$ was considered statistically significant.

\section{Results}

IL-9R is constitutively expressed in HCC cells and tissues. IL-9R has been reported to be expressed in T cell-derived subsets, especially Th2 and Th17 cells (19). Expression of IL-9R was significantly higher in T-cell lymphoma cells than that in normal thymocytes, leading to aberrant downstream STAT3 and STAT5 phosphorylation (24). In the present study, we used western blot and RT-qPCR analyses to determine whether IL-9R was expressed in HCC cells and tissues. RT-qPCR showed that IL-9R was constitutively expressed in $\mathrm{HCC}$ cell lines and that the expression level correlated with HCC cell line metastatic ability (Fig. 1A). Additionally, we found the ligand for IL-9R, such as IL-9, was expressed in HCC cell lines at the mRNA level (data not shown). Western blot analysis validated RT-qPCR results and again indicated that IL-9R was expressed on HCC cells (Fig. 1B). Furthermore, expression level of IL-9R and IL-9 was significantly higher in tumor tissues compared to peritumor tissues by means of RT-qPCR analysis (Fig. $1 C$ and D, $\mathrm{P}<0.001$ ).

IL-9R knockdown mediated by siRNA significantly inhibits cell proliferation. IL-9 stimulates the proliferation of human T-ALL, Hodgkin lymphoma and T-cell leukemic cells (19). To examine the biological function of IL-9R in HCC cells, MHCC97H and MHCC97L were selected for transfection with siRNA duplexes against IL-9R. Successful knockdown in MHCC97H and MHCC97L was confirmed by RT-qPCR (Fig. 2A, P<0.001) and 
A

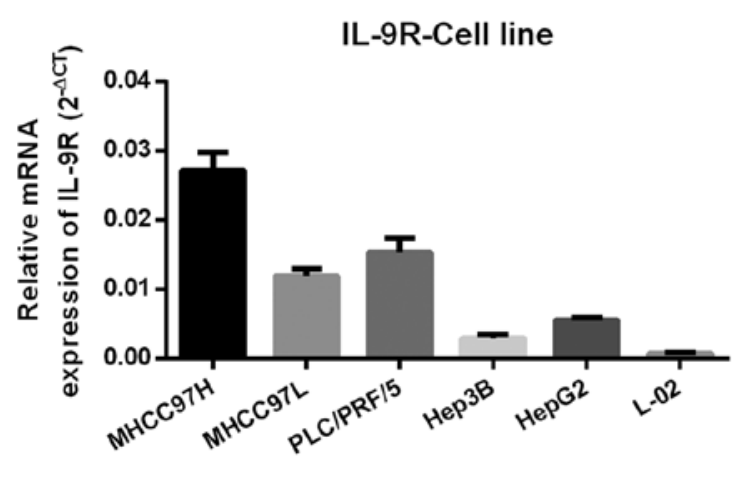

C

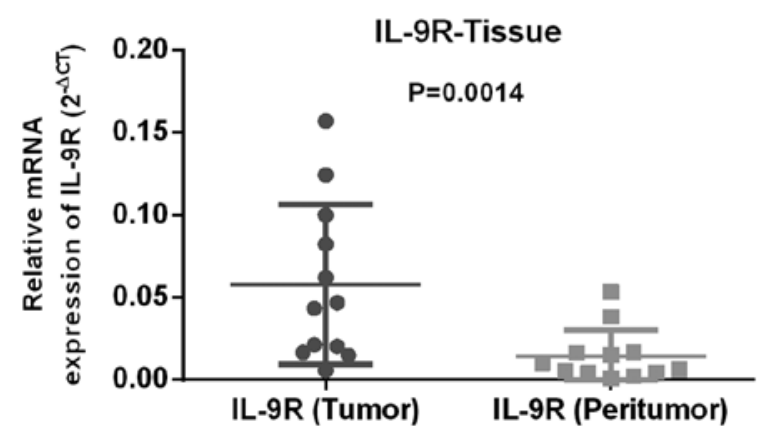

B

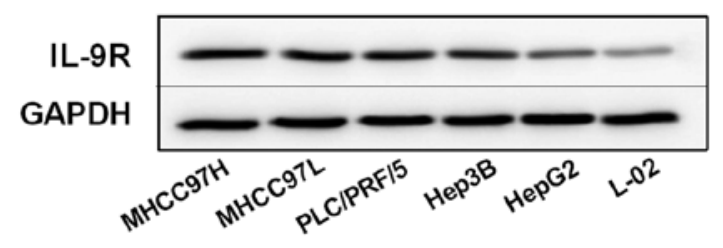

D

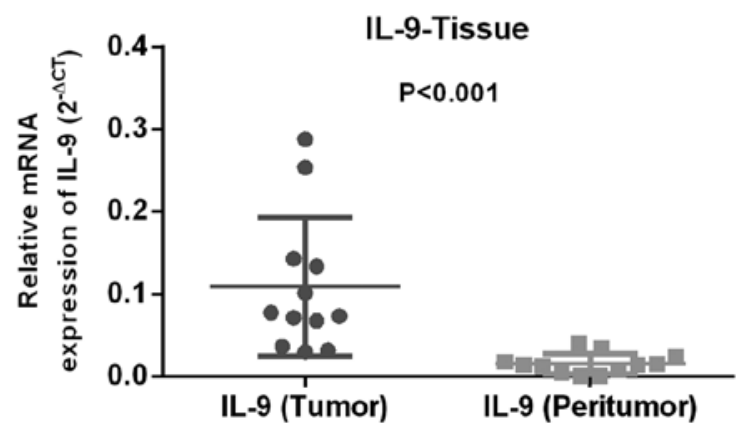

Figure 1. IL-9R constitutively expressed in HCC cells and tissues. (A) RT-qPCR was used to detect IL-9R mRNA expression level in five HCC cells and normal liver L-02 cell lines. (B) Western blot analysis was used to detect the IL-9R protein expression level in the six cell lines. (C) RT-qPCR indicated that IL-9R mRNA expression level was significantly higher in tumor tissues than that in peritumor tissues $(\mathrm{P}=0.0014$, Student's t-test). (D) RT-qPCR detected that the IL-9 mRNA expression level in the tumor tissues was higher than that in the peritumor tissues $(\mathrm{P}<0.001$, Student's t-test). The experiments were performed three times in triplicate.

western blot analysis (Fig. 2B). IL-9R downregulation mediated by siRNA significantly inhibited MHCC97H and MHCC97L cell proliferation following transfection at $72 \mathrm{~h}$ (Fig. 2C and D, $\mathrm{P}<0.001)$. An Annexin V-FITC apoptosis detection kit was used to examine the IL-9R knockdown-mediated effects on cell apoptosis. After IL-9R silencing, the percentage of cells in $\mathrm{S}$ phase markedly decreased, which was consistent with our proliferation analysis showing that inhibition of IL-9R attenuated HCC cell proliferation.

IL-9R downregulation significantly reduces invasiveness and promoted apoptosis of HCC cells. IL-9 is a versatile cytokine with various functions in different types of cells. Previous study of IL-9R in leukemia has suggested that overexpression of IL-9R inhibited leukemic cell apoptosis (18). In the present study, we used a Transwell assay and Annexin V-FITC apoptosis detection kit to determine the biological effect of IL-9R on HCC cells. In the Transwell experiment, MHCC97H and MHCC97L cells invading through the membrane decreased significantly after IL-9R silencing by siRNA (Fig. $3 \mathrm{~A}$ and $\mathrm{B}, \mathrm{P}<0.01$ ). Furthermore, in the apoptosis experiment, the percentage of apoptotic cells increased significantly in the IL-9R-silenced groups compared to the control (Fig. 3C and D, $\mathrm{P}<0.01$ ).

Molecular mechanism involved in IL-9R signaling pathway. IL-9 mediated signal transduction activated molecular members of STAT, MAPK and PI3K pathways (8). In the present study, we analyzed the expression of VEGF, p-p38, p-JNK, p-STAT3,
p-STAT5, STAT5, p-ERK and MMP9, which were previously reported to be potentially involved in the activity of the IL-9/IL9R axis $(8,11,25)$. After the significant downregulation of IL-9R, western blot analysis indicated that the expression of VEGF, p-p38, p-STAT3 and MMP9 obviously decreased (Fig. 3E and F). However, no difference was found in the expression of p-JNK, p-STAT5 and p-ERK (data not shown) following IL-9R downregulation.

High IL-9R expression significantly correlates with reduced survival and early recurrence. Tissue microarrays containing 329 patients were used to evaluate the prognostic value of IL-9R in HCC. Patients with a high (score between 0 and 3, $\mathrm{n}=84$ ) or low (score between 4 and 9, $\mathrm{n}=245$ ) IL-9R expression were divided into two groups. Comparisons were determined between various clinicopathologic characteristics and IL-9R expression (Table I). The correlation analysis showed that a high IL-9R expression significantly correlated with larger tumor $(\mathrm{P}=0.012)$ and advanced TNM stage $(\mathrm{P}=0.018$, Table I). Immunohistochemical staining of IL-9R in HCC cells exhibited a focal or scattered pattern, with different intensity and percentage of staining tumor cells (Fig. 4A).

For the entire cohort $(n=329)$, the OS and RFS rates at 3 , 5 and 7 years of age were $69.60,61.10$ and 56.23, and 72.34, 66.26 , and $59.27 \%$, respectively. In the univariate analysis, liver cirrhosis, $\gamma$-GT, tumor size, tumor differentiation, vascular invasion, TNM stage and BCLC stage were shown to be prognostic factors for OS and RFS (Table II). Additionally, 
A

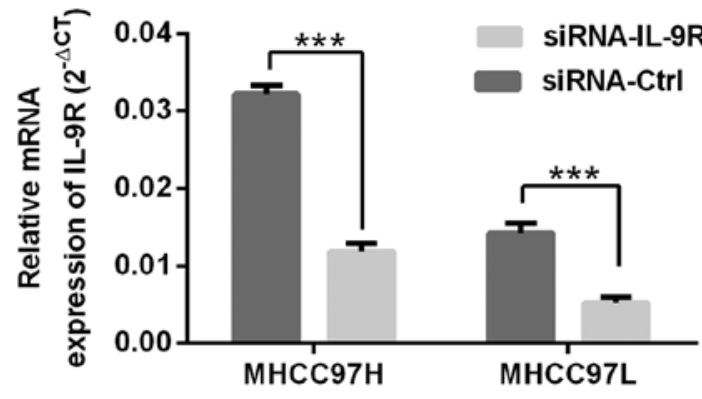

C

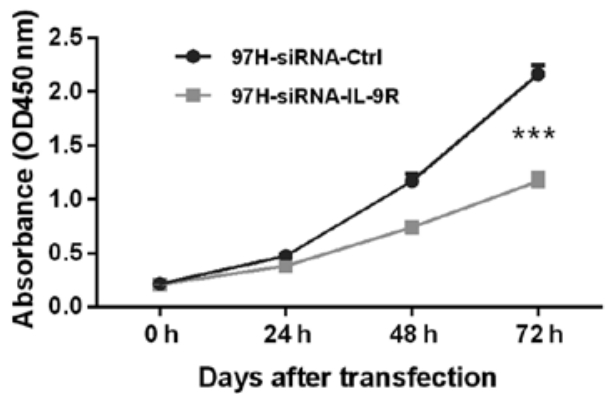

E

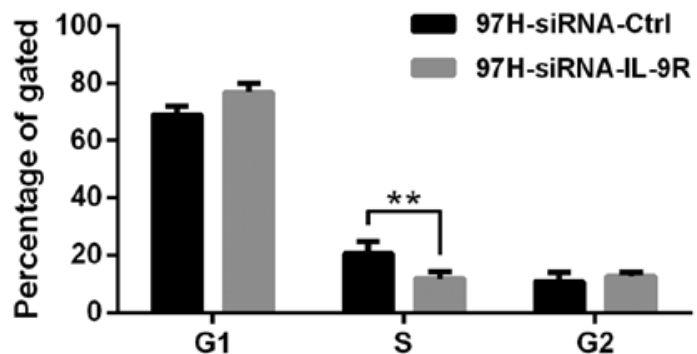

B

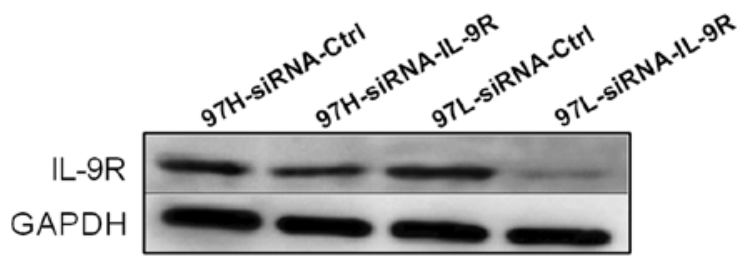

D

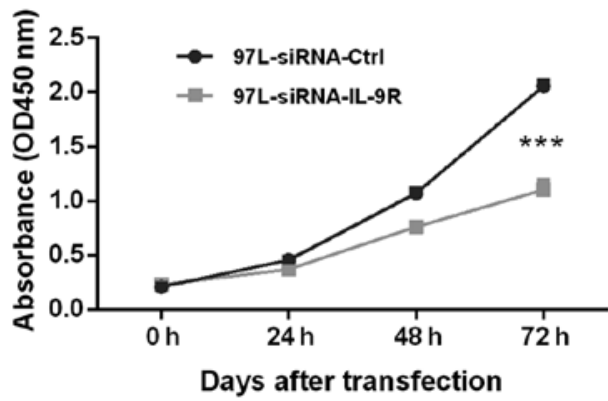

F

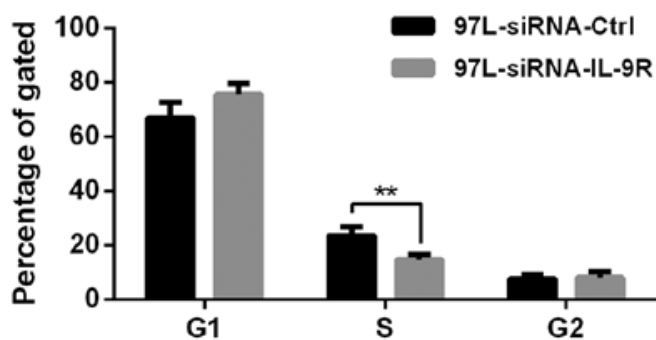

Figure 2. Biological effects of IL-9R on HCC cells. (A and B) RT-qPCR and western blot analysis validated siRNA-IL-9R successfully silenced IL-9R expression. The expression of IL-9R decreased significantly after transfection with siRNA-IL9R. (C and D) After transfection with siRNA-IL9R, absorbance (OD450 nm) values in MHCC97H and MHCC97L significantly decreased at $72 \mathrm{~h}$ compared to the siRNA control groups using a CCK-8 kit. (E and F) Knockdown of IL-9R, cell percentages of MHCC97H and MHCC97L in the S-phase markedly decreased, which was consistent with our proliferation results. $\left({ }^{* *} \mathrm{P}<0.01\right.$ and ${ }^{* * * *} \mathrm{P}<0.001$, Student's t-test). All the experiments were performed in triplicate.

the univariate analysis showed IL-9R expression was a significant prognostic factor for OS $(\mathrm{P}=0.003)$ and RFS $(\mathrm{P}=0.007$, Table II). The multivariate analysis was used to determine whether IL-9R was an independent factor for HCC. Significant factors in the univariate analysis were selected for multivariate analysis. IL-9R was shown to be an independent prognostic factor for OS $(\mathrm{P}=0.005)$ and RFS $(\mathrm{P}=0.030$, Table II $)$. Patients with a high IL-9R expression had significantly decreased OS ( $\mathrm{P}=0.002$, Fig. 4B) and RFS ( $\mathrm{P}=0.018$, Fig. 4C). HCC patients with high IL-9R expression were 1.66 -fold more likely to succumb [95\% CI, 1.17-2.36; $\mathrm{P}=0.005$ ] and 1.5-fold more likely to experience recurrence $(95 \% \mathrm{CI}, 1.04-2.17 ; \mathrm{P}=0.03)$ than the low IL-9R expression patients (Table II).

\section{Discussion}

IL-9 is a versatile cytokine that functions in immune and inflammatory responses as well as in growth-promoting and anti-apoptotic activities suggesting its multifunctional role in tumorigenesis (19). In mast cells, IL-9 acted as a growth factor while in human B cells, IL-9 promoted the production of IgE and IgG through IL-4 signaling (5). The abnormal expression of IL-9R significantly promotes T-ALL cells and inhibits apoptosis in chronic leukemia $(5,25)$.

In the present study, we found that IL-9R was constitutively expressed in the HCC cell lines and tissues of HCC patients. mRNA and protein expression results in the HCC cell lines showed that a high expression of IL-9R correlated with a higher metastatic potential of the cell line. Previous findings showed that there was an abnormal feedback loop of IL-9 and IL-9R in various human leukemias, including T-cell leukemia, megakaryoblastic leukemia and Hodgkin lymphomas $(19,26,27)$. The RT-qPCR detection experiment in this study showed that IL-9R was overexpressed in HCC tumor tissues and the mRNA expression level of ligand IL-9 was significantly higher in tumors than that in peritumors, which indicated a potential feedback loop existed in HCC and it seems to be an active mechanism for HCC tumorigenesis. 
A

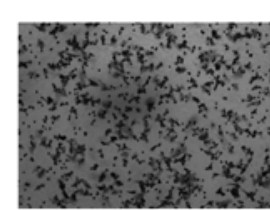

97H-siRNA-Ctrl

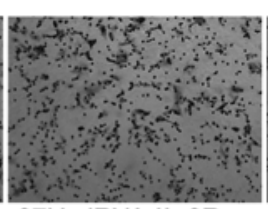

97H-siRNA-IL-9R

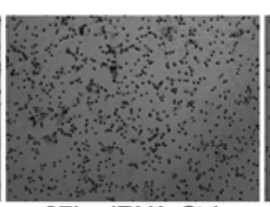

97L-siRNA-Ctr

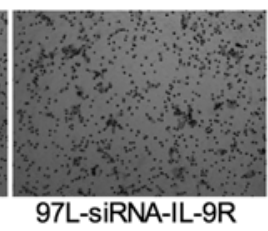

97L-SiRNA-IL-9R
B

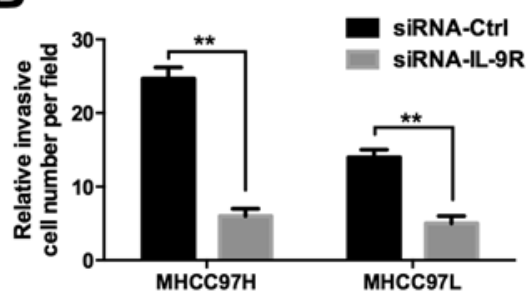

C

D
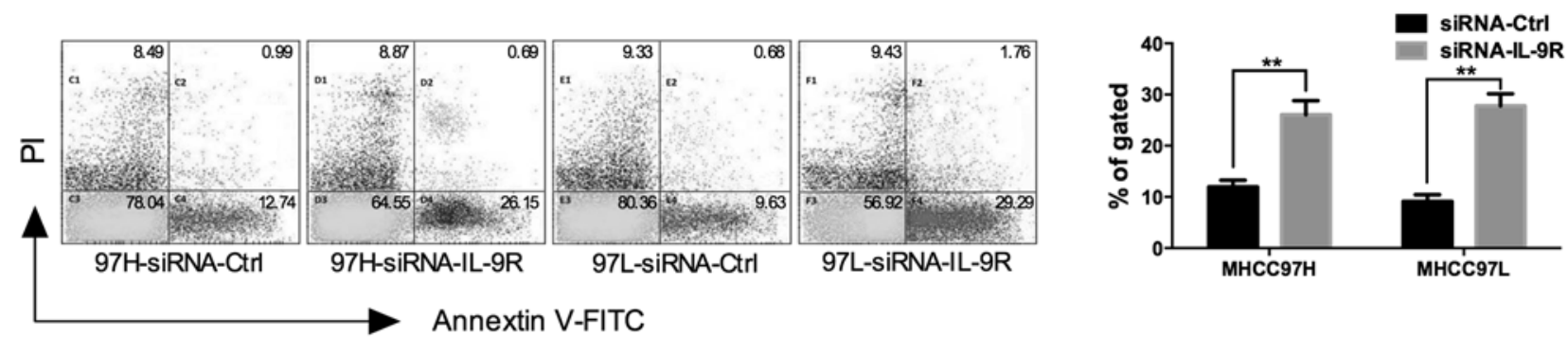

E

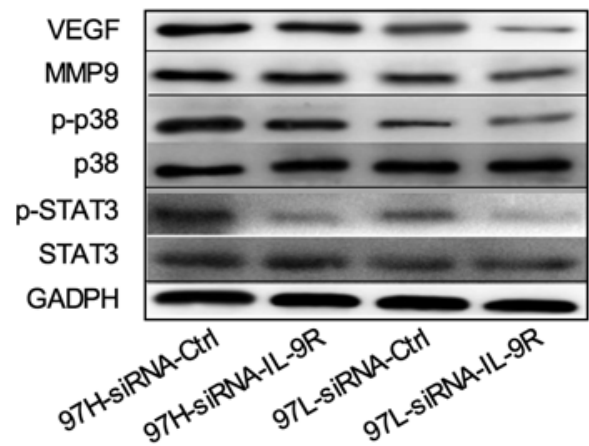

$\mathbf{F}$

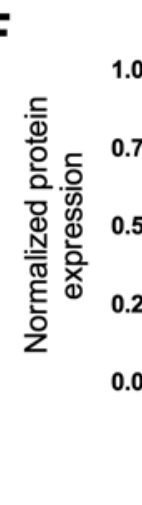

977-Ctrl 97H-siRNA V7. 97L-CtrI W 97L-siRNA

Figure 3. Role of IL-9R in HCC tumorigenesis and molecules involved in IL-9R signaling pathway. (A) A Transwell assay was used to determine HCC cell invasive ability. (B) After downregulation mediated by siRNA, the number of cells migrating through the membrane significantly decreased compared to the control groups. (C) An Annexin V-FITC apoptosis detection kit was used to explore IL-9R-knockdown mediated effects on apoptosis. (D) Percentage of apoptotic cells increased significantly in IL-9R-silenced groups compared to the control. (E and F) Western blot analysis and normalized protein expression indicated that the expression of VEGF, p-p38, p-STAT3 and MMP9 decreased after IL-9R of cell lines was silenced by siRNA. (** P $<0.01$, Student's t-test). The experiments were performed three times in triplicate.

IL-9R was found to be constitutively expressed in HCC tumor cells. We then used proliferation, cell cycle, apoptosis and Transwell assays to determine the biological effect of IL-9R on HCC cells. Downregulation of IL-9R by siRNA in MHCC97H and MHCC97L significantly inhibited cell proliferation compared to the control groups. The percentages of cells in the S-phase were markedly lower than that in the control groups. Results of the two experiments indicated that overexpression of IL-9R in HCC cells promoted cell proliferation. This finding was consistent with previous findings showing that IL-9 promoted the proliferation of T-ALL cells (26), Hodgkin lymphoma cells (28) and natural killer/T-cell lymphoma cells $(19,29)$. It has been shown that IL-9 inhibited apoptosis of the human CLL cell line MEC-1 (18). In the present study, we showed that IL-9R downregulation significantly promoted cell apoptosis and inhibited invasive potential of HCC cell lines. Our in vivo analysis simultaneously demonstrated that a high IL-9R expression significantly correlated with larger tumor volume and advanced TNM stages.

Activation of IL-9R leads to the downstream activation of STAT3, MAPK and PI3K pathway $(8,12,25)$. Additionally, the function of JNK and p38 MAPK family members had a pivotal role in affecting tumor cell proliferation, differentiation and migration (30). Consistent with the importance of these facts, our western blot analysis indicated that after silencing IL-9R, the expression of VEGF, p-p38, p-STAT3 and MMP9 was significantly decreased. Involvement of the four molecules may be the underlying mechanism of the biological effects of IL-9R in HCC cells. By contrast, p-JNK, p-STAT5 or p-ERK did not show any significant changes. Future studies are needed to elaborate the detailed signaling pathways and the exact mechanism involved.

Apart from the tumor-promoting potential of IL-9R, we found that IL-9R was an independent predictor for HCC patients. A cohort of $329 \mathrm{HCC}$ patients indicated that the OS and RFS in the high IL-9R expression group was significantly lower than that in the low expression patients. Patients with a high IL-9R expression were predisposed to succumbing to the disease (1.66; 95\% CI, 1.17-2.36; $\mathrm{P}=0.005)$ and relapse (1.50; 95\% CI, 1.04-2.17; P=0.03).

In conclusion, to the best of our knowledge, we report for the first time the biological and tumor-promoting role of 
Table II. Univariate and multivariate analyses of factors associated with survival and recurrence.

\begin{tabular}{|c|c|c|c|c|c|c|}
\hline \multirow[b]{2}{*}{ Variables } & \multirow{2}{*}{$\begin{array}{c}\text { Univariate } \\
\text { P-value }\end{array}$} & \multicolumn{2}{|c|}{$\begin{array}{l}\text { Overall survival } \\
\text { Multivariate }\end{array}$} & \multirow{2}{*}{$\begin{array}{l}\text { Univariate } \\
\text { P-value }\end{array}$} & \multicolumn{2}{|c|}{$\begin{array}{c}\text { Relapse-free survival } \\
\text { Multivariate }\end{array}$} \\
\hline & & $\mathrm{HR}(95 \% \mathrm{CI})$ & P-value & & $\mathrm{HR}(95 \% \mathrm{CI})$ & P-value \\
\hline Age, year (>52 vs. $\leq 52$ ) & 0.788 & NA & & 0.580 & NA & \\
\hline Gender (male vs. female) & 0.104 & NA & & 0.172 & NA & \\
\hline HBsAg (positive vs. negative) & 0.378 & NA & & 0.306 & NA & \\
\hline Liver cirrhosis (yes vs. no) & 0.012 & $1.39(1.11-1.75)$ & 0.004 & 0.002 & $1.97(1.19-3.30)$ & 0.009 \\
\hline HCC family history (yes vs. no) & 0.375 & NA & & 0.974 & NA & \\
\hline AFP, ng/ml (>20 vs. $\leq 20)$ & 0.141 & NA & & 0.931 & NA & \\
\hline ALT, U/L (>75 vs. $\leq 75)$ & 0.427 & NA & & 0.281 & NA & \\
\hline$\gamma-\mathrm{GT}, \mathrm{U} / \mathrm{L}(>54$ vs. $\leq 54)$ & 0.008 & $1.59(1.02-2.48)$ & 0.040 & 0.245 & NA & \\
\hline Tumor size (>5 vs. $\leq 5)$ & $<0.001$ & $2.21(1.57-3.11)$ & $<0.001$ & $<0.001$ & $1.97(1.19-3.30)$ & $<0.001$ \\
\hline Tumor number (multiple vs. single) & 0.093 & NA & & 0.163 & NA & \\
\hline Tumor capsule (yes vs. no) & 0.105 & NA & & 0.826 & NA & \\
\hline Tumor differentiation (III-IV vs. I-II) & 0.009 & NS & & 0.022 & NS & \\
\hline Vascular invasion (yes vs. no) & 0.001 & $1.57(1.15-2.20)$ & 0.010 & 0.025 & NS & \\
\hline TNM stage (II/III vs. I) & $<0.001$ & NA & & 0.013 & NA & \\
\hline BCLC stage (B/C vs. $0 / A)$ & $<0.001$ & NA & & 0.021 & NA & \\
\hline IL-9R (high vs. low) & 0.003 & $1.66(1.17-2.36)$ & 0.005 & 0.007 & $1.50(1.04-2.17)$ & 0.030 \\
\hline
\end{tabular}

HBsAg, hepatitis B surface antigen; AFP, $\alpha$-fetoprotein; ALT, alanine aminotransferase; $\gamma$-GT, gamma-glutamyl transferase; TNM, tumornode-metastasis; BCLC, Barcelona clinic liver cancer.
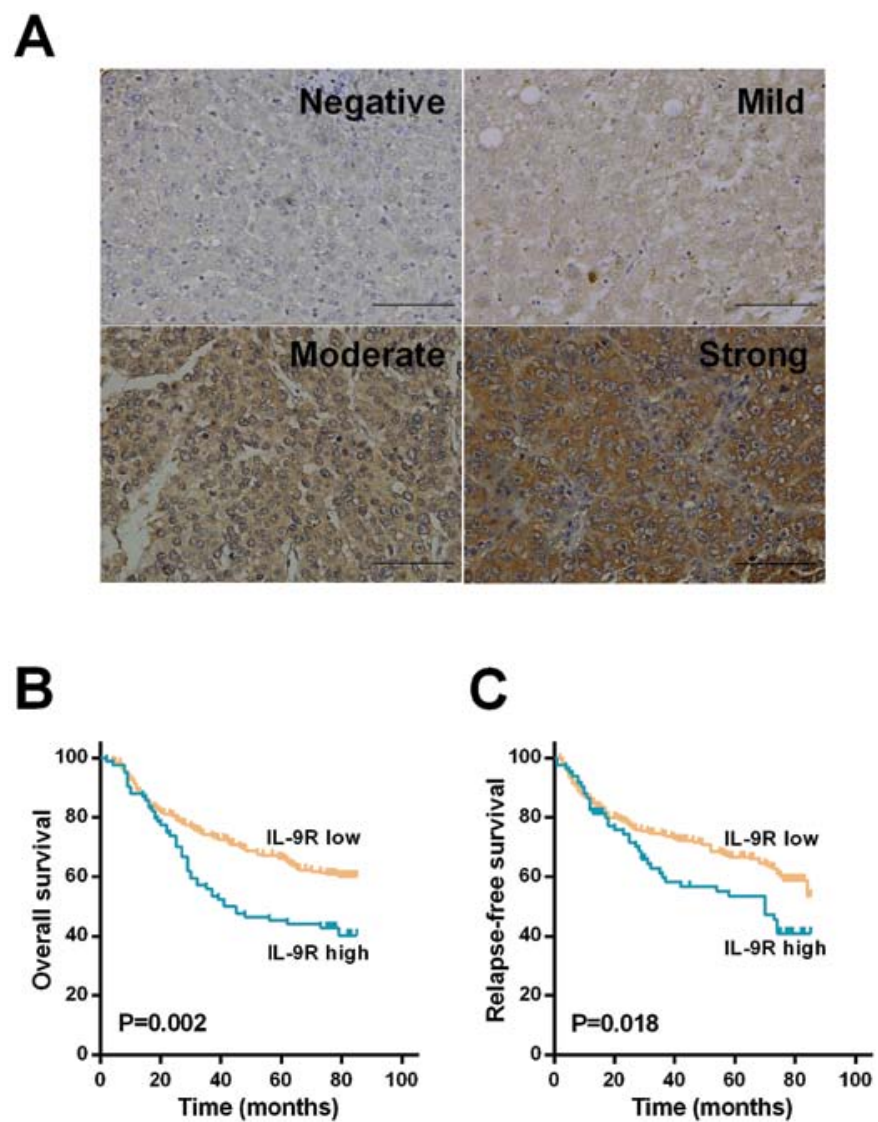

Figure 4. Overexpression of IL-9R significantly correlated with poorer survival. (A) Immunohistochemistry was used to evaluate the correlation between IL-9R expression and prognosis of HCC patients. IL-9R in HCC cells exhibited a focal or scattered pattern, with different intensity and the percentage of staining tumor cells. (B and C) We used Kaplan-Meier method and log-rank test to evaluate OS and RFS difference between IL-9R high expression and low expression patients. Patients with a high IL-9R expression had significantly poorer OS $(\mathrm{P}=0.002)$ and $\mathrm{RFS}(\mathrm{P}=0.018, \log -\mathrm{rank}$ test $)$. 
IL-9R in HCC. IL-9R was constitutively expressed in HCC cells, promoted cell proliferation and invasive ability, inhibited apoptosis and acted as an independent predictor for the survival of HCC. Therefore, together with our findings, IL-9R may be used as a potential biomarker in HCC therapy.

\section{References}

1. Jemal A, Bray F, Center MM, Ferlay J, Ward E and Forman D Global cancer statistics. CA Cancer J Clin 61: 69-90, 2011.

2. Forner A, Llovet JM and Bruix J: Hepatocellular carcinoma. Lancet 379: 1245-1255, 2012.

3. Niu ZS, Niu XJ and Wang M: Management of hepatocellular carcinoma: Predictive value of immunohistochemical markers for postoperative survival. World J Hepatol 7: 7-27, 2015.

4. Hanash SM, Baik CS and Kallioniemi O: Emerging molecular biomarkers - blood-based strategies to detect and monitor cancer. Nat Rev Clin Oncol 8: 142-150, 2011.

5. Stassen M, Schmitt E and Bopp T: From interleukin-9 to T helper 9 cells. Ann N Y Acad Sci 1247: 56-68, 2012.

6. Renauld JC, Druez C, Kermouni A, Houssiau F, Uyttenhove C, Van Roost E and Van Snick J: Expression cloning of the murine and human interleukin 9 receptor cDNAs. Proc Natl Acad Sci USA 89: 5690-5694, 1992.

7. Noelle RJ and Nowak EC: Cellular sources and immune functions of interleukin-9. Nat Rev Immunol 10: 683-687, 2010.

8. Goswami R and Kaplan MH: A brief history of IL-9. J Immunol 186: 3283-3288, 2011.

9. Demoulin JB and Renauld JC: Interleukin 9 and its receptor: An overview of structure and function. Int Rev Immunol 16 : 345-364, 1998.

10. Druez C, Coulie P, Uyttenhove C and Van Snick J: Functional and biochemical characterization of mouse P40/IL-9 receptors. J Immunol 145: 2494-2499, 1990.

11. Demoulin JB, Uyttenhove C, Van Roost E, DeLestré B, Donckers D, Van Snick J and Renauld JC: A single tyrosine of the interleukin-9 (IL-9) receptor is required for STAT activation, antiapoptotic activity, and growth regulation by IL-9. Mol Cell Biol 16: 4710-4716, 1996.

12. Demoulin JB, Louahed J, Dumoutier L, Stevens M and Renauld JC: MAP kinase activation by interleukin-9 in lymphoid and mast cell lines. Oncogene 22: 1763-1770, 2003.

13. Yamasaki A, Saleh A, Koussih L, Muro S, Halayko AJ and Gounni AS: IL-9 induces CCL11 expression via STAT3 signalling in human airway smooth muscle cells. PLoS One 5: e9178, 2010.

14. Jäger A, Dardalhon V, Sobel RA, Bettelli E and Kuchroo VK: Th1, Th17, and Th9 effector cells induce experimental autoimmune encephalomyelitis with different pathological phenotypes. J Immunol 183: 7169-7177, 2009.

15. Forbes EE, Groschwitz K, Abonia JP, Brandt EB, Cohen E, Blanchard C, Ahrens R, Seidu L, McKenzie A, Strait R, et al: IL-9- and mast cell-mediated intestinal permeability predisposes to oral antigen hypersensitivity. J Exp Med 205: 897-913, 2008.

16. Sismanopoulos N, Delivanis DA, Alysandratos KD, Angelidou A, Vasiadi M, Therianou A and Theoharides TC: IL-9 induces VEGF secretion from human mast cells and IL-9/IL-9 receptor genes are overexpressed in atopic dermatitis. PLoS One 7: e33271, 2012.
17. Kearley J, Erjefalt JS, Andersson C, Benjamin E, Jones CP, Robichaud A, Pegorier S, Brewah Y, Burwell TJ, Bjermer L, et al: IL-9 governs allergen-induced mast cell numbers in the lung and chronic remodeling of the airways. Am J Respir Crit Care Med 183: 865-875, 2011.

18. Chen N, Lu K, Li P, Lv X and Wang X: Overexpression of IL-9 induced by STAT6 activation promotes the pathogenesis of chronic lymphocytic leukemia. Int J Clin Exp Pathol 7: 2319-2323, 2014

19. Shang Y, Kakinuma S, Nishimura M, Kobayashi Y, Nagata K and Shimada Y: Interleukin-9 receptor gene is transcriptionally regulated by nucleolin in T-cell lymphoma cells. Mol Carcinog 51: 619-627. 2012

20. El-Serag HB: Epidemiology of viral hepatitis and hepatocellular carcinoma. Gastroenterology 142: 1264-1273.e1, 2012.

21. Shi JY, Gao Q, Wang ZC, Zhou J, Wang XY, Min ZH, Shi YH, Shi GM, Ding ZB, Ke AW et al: Margin-infiltrating CD20(+) $B$ cells display an atypical memory phenotype and correlate with favorable prognosis in hepatocellular carcinoma. Clin Cancer Res 19: 5994-6005, 2013.

22. Vitale A, Morales RR, Zanus G, Farinati F, Burra P, Angeli P, Frigo AC, Del Poggio P, Rapaccini G, Di Nolfo MA, et al: Italian Liver Cancer group: Barcelona Clinic Liver Cancer staging and transplant survival benefit for patients with hepatocellular carcinoma: A multicentre, cohort study. Lancet Oncol 12: 654-662, 2011.

23. Gao Q, Zhao YJ, Wang XY, Qiu SJ, Shi YH, Sun J, Yi Y, Shi JY, Shi GM, Ding ZB, et al: CXCR6 upregulation contributes to a proinflammatory tumor microenvironment that drives metastasis and poor patient outcomes in hepatocellular carcinoma. Cancer Res 72: 3546-3556, 2012.

24. Shang Y, Kakinuma S, Amasaki Y, Nishimura M, Kobayashi Y and Shimada Y: Aberrant activation of interleukin-9 receptor and downstream Stat3/5 in primary T-cell lymphomas in vivo in susceptible B6 and resistant $\mathrm{C} 3 \mathrm{H}$ mice. In Vivo 22: 713-720, 2008.

25. Chen N and Wang X: Role of IL-9 and STATs in hematological malignancies (Review). Oncol Lett 7: 602-610, 2014.

26. Barata JT, Keenan TD, Silva A, Nadler LM, Boussiotis VA and Cardoso AA: Common gamma chain-signaling cytokines promote proliferation of T-cell acute lymphoblastic leukemia. Haematologica 89: 1459-1467, 2004.

27. Glimelius I, Edström A, Amini RM, Fischer M, Nilsson G, Sundström C, Enblad G and Molin D: IL-9 expression contributes to the cellular composition in Hodgkin lymphoma. Eur J Haematol 76: 278-283, 2006.

28. Gruss HJ, Brach MA, Drexler HG, Bross KJ and Herrmann F: Interleukin 9 is expressed by primary and cultured Hodgkin and Reed-Sternberg cells. Cancer Res 52: 1026-1031, 1992.

29. Nagato T, Kobayashi H, Kishibe K, Takahara M, Ogino T, Ishii H, Oikawa K, Aoki N, Sato K, Kimura S, et al: Expression of interleukin-9 in nasal natural killer/T-cell lymphoma cell lines and patients. Clin Cancer Res 11: 8250-8257, 2005.

30. Wagner EF and Nebreda AR: Signal integration by JNK and p38 MAPK pathways in cancer development. Nat Rev Cancer 9 : 537-549, 2009. 